\title{
The response of denervated muscle to long-term electrical stimulation
}

\author{
T. Lømo, R.H. Westgaard, R. Hennig, K. Gundersen
}

Institute of Neurophysiology, University of Oslo, Karl Johansgate 47, 0162 Oslo 1, Norway

\begin{abstract}
Adapted from: Lømo T, Westgaard RH, Hennig R, Gundersen K. The response of denervated muscle to long-term electrical stimulation, In: Carraro U, Angelini C, eds. Proceedings of the First Abano Terme Meeting on Rehabilitation, 1985 August 28-30, Abano Terme, Padova, Italy, An International Symposium, Satellite Meeting of the XIII World Congress of Neurology, Hamburg 1985. Cleup Padova 1985. pp 81-90.
\end{abstract}

Eur J Trans Myol - Basic Appl Myol 2014; 24 (1): 21-25

The effects of denervation, cross-reinnervation and chronic nerve stimulation show that motoneurons control the properties of skeletal muscles. ${ }^{1-6}$ Evoked muscle activity and neurotrophic substances released from motor nerve terminals may mediate this control, either independently or in combination. However, the two mechanisms are difficult to separate in preparations where the nerve is intact, because changes in neural activity may influence the content or release of neurotrophic substances. We have, therefore, as an extension of earlier work, ${ }^{7,8}$ denervated rat fast and slow muscles to remove putative neurotrophic substances, and then stimulated the muscles electrically with different stimulation patterns to examine the effects of evoked activity per se on extrajunctional membrane properties and contractile properties.

\section{Materials and Methods}

Young, adult, male, Wistar rats, weighing 250-350 grams, were used. The operations were done under barbiturate or ether anesthesia. Soleus and extensor digitorum longus (edl) muscles were denervated by cutting and reflecting the sciatic nerve in the thigh. A pair of Teflon coated multistranded steel wires was implanted. The distal ends, with the insulation removed, were placed across the edl or the soleus muscle, one on each side. And the wires were run under the skin, through an attachment by screws and dental cement on the skull to a stimulator above the rat. The rat was keept in a large bucket, where it could move freely. Stimulation started 1 day to 9 months after denervation, lasted from 1 day to 9 months, and consisted of different trains of stimuli (Table 1). Each stimulus pulse was bipolar. The duration was $0.2 \mathrm{msec}$ in each direction, and the intensity 5-10 mA.

In one series of experiments the muscles were removed from the rat and placed in a chamber superfused with oxygenated Ringer solution at room temperature. Conventional micropipettes filled with $4 \mathrm{M}$ K-acetate or $3 \mathrm{M}$ acetylcholine chloride $(\mathrm{AChCl})$ were then used to record resting membrane potentials (RMP) and sensitivity to $\mathrm{ACh}^{.}{ }^{9}$ In another series the leg was inserted into a Perspex chamber containing oxygenated Ringer solution at $35^{\circ} \mathrm{C}$. The distal part of the soleus or edl was dissected free and connected to a force transducer, while the main blood supply was kept intact. Twitch and tetanic contractions, evoked by direct supra maximal stimuli from large platinum electrodes, were then measured under isometric conditions, at optimal length. Lack of reinnervation was confirmed by stimulating the nerve just outside the muscle and looking for muscle contractions with the dissection microscope.

\section{Results}

Fig. 1 A shows first, that denervation causes a striking increase in extrajunctional Ach sensitivity; second, that electrical stimulation abolishes this sensitivity; and third, that stopping the stimulation causes it to reappear. In Fig. 1 B the RMP falls after denervation, and then rises to normal values after the onset of stimulation. Also in denervated edl muscles stimulation removes extrajunctional $\mathrm{ACh}$ sensitivity and restore normal RMPs (not shown).

Fig. 2 shows, first, that denervation causes a striking reduction in force of soleus muscles; second, that electrical stimulation either maintains or restores the force output to nearly normal values.

The cross sectional areas of the muscle fibres undergo parallel changes (not shown). The denervated and stimulated muscles usually produce less force than normal muscles, but so do innervated muscles when stimulated in the same way (Fig. 2). Long-term stimulation causes similar increases in force output and muscle fiber cross sectional area in edl muscles (not shown).

Fig. 3 shows, first, that a fast stimulation pattern (intermittent $100 \mathrm{~Hz}$ ) markedly reduces the twitch contraction time of denervated soleus muscles; second, that denervation and implantation of sham electrodes 


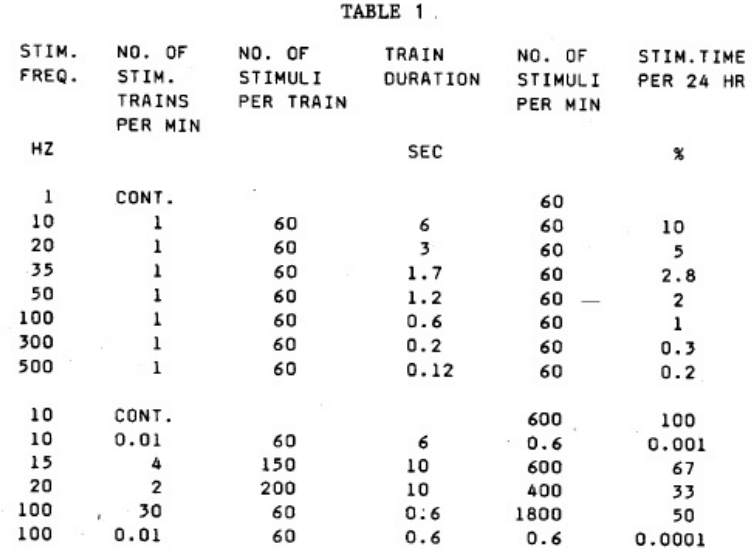

has no comparable speeding up effect; and third, that a slow stimulation pattern (continuous $10 \mathrm{~Hz}$ ) maintains or increases the original contraction time. During fast pattern stimulation the half relaxation time also declines markedly, and the properties of "sag" and post tetanic potentiation, typical of fast muscles, ${ }^{9,10}$ appear. In contrast, during tonic, low frequency stimulation (10 $\mathrm{Hz}$, mean frequency $10 \mathrm{~Hz}$ ), the twitch contraction time and other contractile properties remain slow.

Fig.4 shows the twitch contraction time of soleus muscles after about 2 months of denervation and stimulation with the stimulus patterns indicated in Table 1. The slowest contraction time (about $45 \mathrm{msec}$ ) is obtained during tonic, low frequency stimulation, and the fastest (about $12 \mathrm{msec}$ ) during intermittent, high frequency stimulation. When the amount of stimulation at a given frequency is reduced, the twitch contraction time is also reduced. For example, at $10 \mathrm{~Hz}$ a 1000 fold reduction in mean stimulus frequency (from 10 to $0.01 \mathrm{~Hz}$ ) reduces the contraction time from $\sim 45$ to $19 \mathrm{msec}$. However, to make the contraction time as fast as in the edl $(\sim 12 \mathrm{msec})$, high frequency stimulation is necessary. By changing both the amount

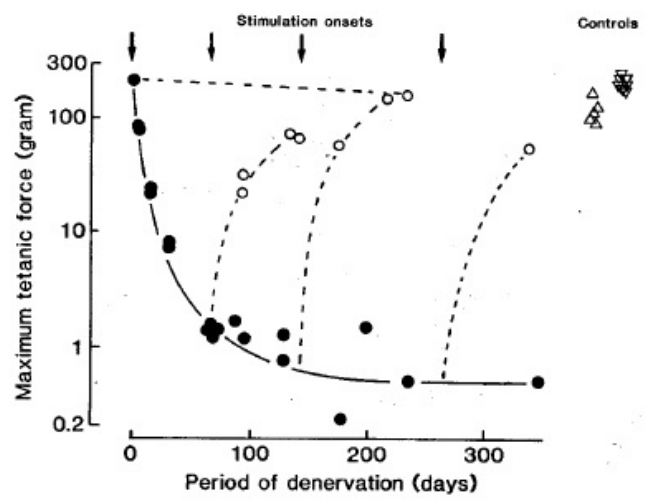

Fig. 2. Maximum tetanic force output of soleus muscles at different times after denervation $(\bullet)$, or denervation and intermittent $100 \mathrm{~Hz}$ stimulation ( $\mathrm{O}$, one train of 60 pulses every $60 \mathrm{sec}$ ). In 4 different experiments the stimulation started at the times indicated by the arrows. represents normal soleus muscles from intact rats. $\Delta$ represents innervated soleus muscles stimulated for about 3 months in the same way as the denervated muscles.
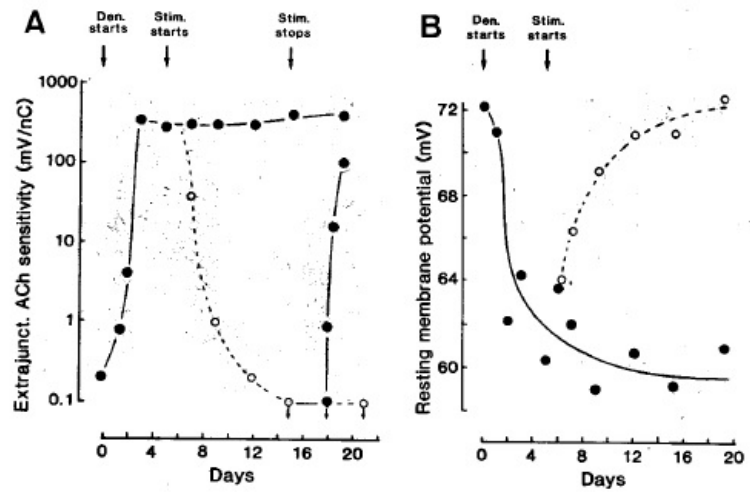

Fig. 1. Extrajunctional Ach sensitivity measured $2 \mathrm{~mm}$ from the tendon ( $A$ ), and resting membrane potential ( $B$ ) of soleus muscle fibres. The muscles were denervated (, stimulated at $100 \mathrm{~Hz}$ (O, one train of 100 pulses every 100 sec), and then stimulation was stopped ( $\bullet$ from the times indicated by the arrows. Symbols represent mean values from many fibres in several muscles. Fro

and the frequency of stimulation it is possible to continuously grade the contraction time within a certain range (45-12 msec), which we call the "adaptive range" for this parameter in the soleus.

Other contractile parameters can also be regulated continuously within certain adaptive ranges. For example, during intermittent high frequency stimulation the intrinsic shortening velocity of the soleus (whole muscle isotonic shortening velocity corrected for differences in fiber length) becomes only half as fast as in the normal edl. ${ }^{11}$ Furthermore, the soleus fibres continue to bind anti-slow myosin, while acquiring the ability to bind anti-fast myosin, i.e. the stimulated soleus fibres show incomplete transformation and hybrid characteristics. $^{11}$ In the denervated edl the adaptive range of time extends from about 12 to $23 \mathrm{msec}$. Neither denervation alone, nor tonic, low frequency stimulation directly after denervation, or indirectly via the intact nerve (reference $^{12}$ and this work), make the contraction time of the edl slower than $23 \mathrm{msec}$.

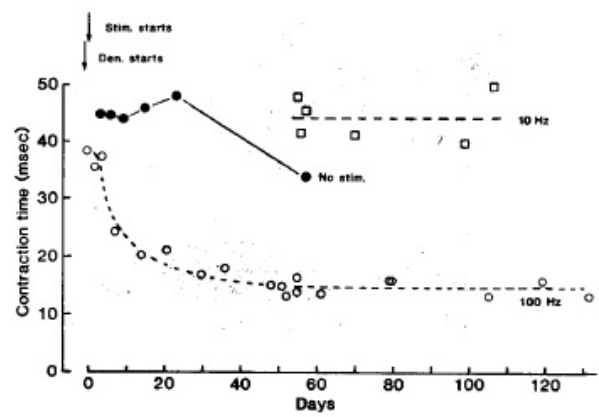

Fig. 3. Isometric twitch contraction time of soleus muscles at different times after denervation and onset of electrical stimulation (arrows). represents the fast stimulation pattern ( 60 pulses at $100 \mathrm{~Hz}$ every min). represents the slow stimulation pattern (continous io $\mathrm{Hz}$ ). represents denervated, non stimulated soleus muscles with sham electrodes implanted. Each is mean of 2 muscles. Some lectrodes of 2 muscles, Some are means of up to 8 muscles. The other symbols represent single muscles. 
The response of denervated muscle to long-term electrical stimulation - 1985

Eur J Trans Myol - Basic Appl Myol 2014; 24 (1): 21-25

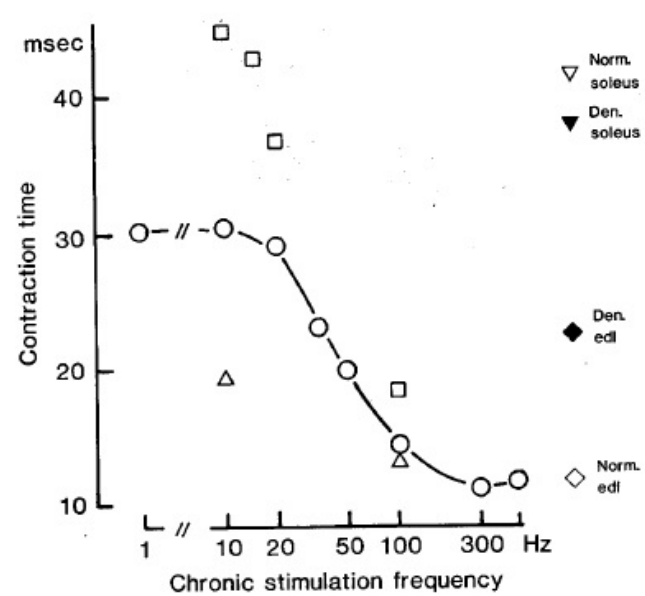

Fig. 4. Isometric twitch contraction times measured about 2 months after denervation and stimulation with the stimulus patterns indicated in Table 1 . represent muscles stimulated once every $m$ in with a train of 60 pulses at the frequencies indicated along the abcissa (mean frequency $1 \mathrm{~Hz}$ ). represent muscles stimulated at $10,15,20$, and $100 \mathrm{~Hz}$ (abcissa), elther continously at $10 \mathrm{~Hz}$ or with large numbers of stimulus trains (mean frequecies of $10,10,6.7$, and $50 \mathrm{~Hz}$ respectively). represent muscles stimulated once every 1 hour and $40 \mathrm{~min}$ with a train of 60 pulses at 10 or $100 \mathrm{~Hz}$ (mean frequency with a train of 60 pulses at 10 or $100 \mathrm{~Hz}$ (mean frequenc
$0.01 \mathrm{~Hz}$ ). Symbols on the right represent normal and denervated control muscles as indicated. Each symbol is the mean of 2-10 muscles.

\section{Discussion}

Long-term electrical stimulation restores normal extrajunctional membrane properties in denervated slow and fast muscles in the absence of intramuscular motor axons and, therefore, in the absence of putative neurotrophic factors. Stimulation also causes a marked, but usually incomplete, recovery of muscle size and force output. The incomplete recovery could easily have a trivial explanation. For example, muscle stretch which is known to affect muscle fiber size, ${ }^{12,13}$ is certainly inadequate in these experiments because the entire rat limb is denervated and the working mode atypical. The electrodes might also damage or fail to activate part of the muscle. Furthermore, some atrophy usually occurs in innervated muscles during long-term electrical stimulation, although, in this case, putative neurotrophic substances should be present (Fig. 2, and references. ${ }^{3,5,6}$ For these reasons, moderate atrophy in denervated and stimulated muscles should not be taken as evidence that essential neurotrophic substances are lacking. On the contrary, the effects of stimulation on the extrajunctional membrane properties, and on the contractile properties of denervated muscles, are so striking that an essential neurotrophic control mechanism seems unlikely in the rat.

If this conclusion can be extended to humans, then it should be possible to maintain and perhaps make some use of denervated muscles in humans by suitable electrical stimulation. If, on the other hand, neurotrophic substances are essential, such prospects seem less likely.
TABLE 2

\begin{tabular}{|c|c|c|c|c|c|}
\hline \multirow[b]{2}{*}{ MUSCLE } & \multicolumn{2}{|c|}{ EXPERIMENTAL PROCEDURE } & \multicolumn{3}{|c|}{ CONTRACTION TIME (MSEC) } \\
\hline & $\begin{array}{l}\text { SURG ICAL } \\
\text { OPERATION }\end{array}$ & $\begin{array}{l}\text { STIMULUUS } \\
\text { PATTERN }\end{array}$ & $\begin{array}{l}\text { CONTROL } \\
\text { MUSCLES }\end{array}$ & $\begin{array}{l}\text { EXPERIM. } \\
\text { MUSCLES }\end{array}$ & REF. \\
\hline SOLEUS & NERVES CROSSED & - & 37.8 & 15.6 & 3 \\
\hline SOLEUS & MUSCLES CROSSED & - & 37.0 & 13.0 & 12 \\
\hline SOLEUS & MUSCLE DENERV. & FAST & 40.4 & 12.0 & \\
\hline SOLEUS & MUSCLE INNERV. & FAST & 39.2 & 22.9 & \\
\hline SOLEUS & MUSCLE DENERV. & $\mathrm{FAST}+\mathrm{SLOW}$ & 40.4 & 22.3 & \\
\hline EDL & NERVES CROQSSED & - & 12.6 & 21.3 & 3 \\
\hline EDL & MUSCLES CROSSED & - & 12.7 & 23.6 & 12 \\
\hline EDL & MUSCLE DENERV. & SLOW & 11.9 & 21.4 & \\
\hline
\end{tabular}

Contraction speed is determined partly by the amount of activity. Thus, fast muscles become slower when stimulated at low or high frequency, ${ }^{3,5,6}$ while slow muscles become faster when the amount of activity is reduced, as after spinal cord section, ${ }^{14,15}$ or immobillzation. ${ }^{16,17}$ Therefore, it has been proposed that slow contraction speeds are due to tonic activity per se, while fast speeds may be due to intrinsic muscle properties ${ }^{6}$ or some neurotrophic factor, ${ }^{18}$ which would make all muscles fast when tonic activity is absent. Accordingly, fast activity patterns are not considered to play a significant role in determining fast twitch properties, ${ }^{19}$ and the fast speed of muscles crossreinnervated with a fast nerve is attributed to the absence of tonic activity rather than the high frequency activity of fast motoneurones. ${ }^{20}$

Our results suggest a different view. Also we find that the soleus can be made considerably faster merely by reducing the amount of activity, particularly at low stimulation frequencies (Fig. 4). However, to obtain contraction times as short as those found in normal edl muscles, or in the soleus after cross-reinnervation, high frequency stimulation is necessary (Fig. 4 and Table 2 ). Furthermore, when high frequency stimulation is added to tonic, low frequency activity, evoked either by electrical stimulation or naturally by an intact soleus nerve, then the contraction speed increases considerably despite the increased amount of activity (Table 2). Therefore, high frequency activity appears specifically to induce fast contractile properties.

Using stimulation patterns comparable to the firing patterns of normal soleus and edl motoneurons of freely moving rats, ${ }^{21}$ we find that the soleus maintains a normal slow contraction time during slow pattern stimulation, and acquires a contraction time as fast as in the normal edl during fast pattern stimulation (Fig. 4 and Table 2). These effects are virtually the same as those obtained by self-reinnervation as those obtained by the slow soleus nerve or cross-innervation by the fast edl nerve (Table 2). Similar results are obtained in the edl, where fast stimulation patterns have the same effects as self-reinnervation by the original fast nerve, and slow stimulation patterns the same effects as crossreinnervation by the slow soleus nerve (Table 2). However, the two muscles respond strikingly different 


\section{The response of denervated muscle to long-term electrical stimulation - 1985}

Eur J Trans Myol - Basic Appl Myol 2014; 24 (1): 21-25

to similar inputs. Thus, the edl acquires a contraction time of only $23 \mathrm{msec}$ during slow pattern stimulation or after slow nerve reinnervation, whereas the soleus acquires a contraction time of $38-40$ msec. This indicates; first, that motoneurons control contraction speed primarily through the patterns of activity that they evoke in the muscle fibres; and second, that the muscle fibres of edl and soleus muscles have developed different intrinsic properties. Such intrinsic differences might explain why similar impulse activities, innervation, ${ }^{2,22,23}$ or hormones, ${ }^{4}$ may have different effects on different types of muscle fibres.

In conclusion, our results indicate; first, that neurally evoked muscle activity plays an essential role in the control of extrajunctional membrane and contractile properties; second, that muscle fibres display adaptive ranges within which a contractile property, such as twitch speed, can be continuously graded by different patterns of impulse activity, and where both the amount and the frequency of impulses are important; and third, that different types of muscle fibres have different adaptive ranges, because of different intrinsic properties. Thus, the fast speed of a rat edl muscle apparently results partly from intrinsic properties and partly from the high frequency activity typical of edl motoneurons, while the slow speed of rat soleus results partly from intrinsic properties that are different from those in edl and partly from the low frequency, tonic activity typical of soleus motoneurons. As a result contractile properties may be adapted to the varying functional demands imposed by the central nervous system within the characteristic adaptive range of each muscle fiber.

\section{Corresponding Author}

Terje Lømo (MD, PhD), Institute of Basic Medical Sciences, University of Oslo, Norway

E-mail: terje.lomo@basalmed.uio.no

\section{References}

1. Buller AJ, Eccles JC, Eccles RM. Interactions between motoneurons and muscles in respect of the characteristic speeds of their responses. J Physiol 1960;150:417-39.

2. Close R. Dynamic properties of fast and slow muscles of the rat after nerve cross-union. J Physiol 1969;204:331-46.

3. Eerbeek 0, Kernell D, Verhey BA. Effects of fast and slow patterns of tonic long-term stimulation on contractile properties of fast muscle in the cat. J Physiol 1984;352:73-90.

4. Gutmann E. Neurotrophic relations. Ann Rev Physiol 1976;38:177-216.

5. Pette 0 , Müller W, Leisner WE, Vrbovà G. Time dependent effects on contractile properties, fibre population, myosin light chains end enzymes of energy metabolism in intermittently and continously stimulated fast twitch muscles of the rabbit. Plügers Arch 1976;364:103-12.

6. Salmons S, Sreter FA. Significance of impulse activity in the transformation of skeletal muscle type. Nature 1976;263:30-4.

7. Lømo T, Westgaard RH, Dahl HA. Contractile properteies of muscle: control by pattern of muscle activity in the rat. Proc Roy Soc B 1974;187:99-103.

8. Lømo T, Westgaard RH. Control of ACh sensitivity in rat muscle fibres. Cold Spring Harbor Symp Quant Biol 1976;60:263-74.

9. Lømo T, Westgaard RH. Further studies on the control of ACh sensitivity by muscle activity in the rat. J Physiol 1975;252:603-26.

10. Burke RE, Levine DN, Tsairis P, Zajac FE. Physiological types and histochemical profiles in motor units of the cat gastrocnemius. J Physiol 1973;234:723-48.

11. Close R, Hoh JFY. Post-tetanic potentiation of twitch contractions of cross-innervated rat fast and slow muscles. Nature 1969;221:179-81.

12. Gorza L, Gundersen K, Lømo T, et al. Transformation of slow to fast contractile properties during chronic stimulation of rat soleus muscles. In preparation.

13. Kwong WH, and Vrbovà G. Effect of lowfrequency electrical stimulation on fast and slow muscles of the rat. Plügers Arch 1981;391:200-7.

14. Frankeny JR, Holly RG, Ashmore CR. Effects of graded durations of stretch on normal and dystrophic skeletal muscle. Muscle Nerve 1983; 4:269-77.

15. Vandenburgh, HH, Kaufman S. In vitra skeletal muscle hypertrophy and $\mathrm{Na}$ pump activity. In: Plasticity of Muscle. Pette D, Ed. Berlin, New York. de Gruyter, 1980 pp. 494-506.

16. Hoh JFY, Kwan BTS, Dunlop C, Kim BH. Effects of nerve cross-union and cordotomy on myosin isoenzymes in fast-twitch and slow-twitch muscles of the rat. In: Plasticity of muscle. Pette D, Ed. Berlin, New York: de Gruyter, 1980 pp. 339-52.

17. Fischbach GD, Robbins N. Changes in contractile properties of disused soleus muscles. J Physiol 1969;201:305-20.

18. Mayer, RF,Burke RE, Toop J. The effect of longterm immobilization on the motor unit population of the cat medial gastrocnemius muscle. Neuroscience 1981;4:725-39.

19. Mayer RF, Burke RE, Toop, J, et al. The effect of spinal cord transection on motor units in cat medial gastrocnemius muscle. Muscle Nerve 1984;7:23-31.

20. Gallego R, Huizar P, Kudo N, Kuno M. Disparity of motoneurone and muscle differentiation following spinal transection in the kitten. J Physiol 1978;281:253-65. 
The response of denervated muscle to long-term electrical stimulation - 1985

Eur J Trans Myol - Basic Appl Myol 2014; 24 (1): 21-25

21. Jolesz F, Sreter FA. Development, innervation, end activity-pattern induced changes in skeletal muscle. Ann Rev Physiol 1981;43:531-52.

22. Salmons S, Henriksson J. The adaptive response of skeletal muscle to increased use. Muscle Nerve 1981;4:94-105.

23. Hennig R, Lømo T. Firing patterns of motor units in normal rats. Nature 1985;314:164-6.
24. Gauthier GF, Burke RE, Lowey S, Hobbs AW. Myosin isoenzymes in normal and cross reinnervated cat skeletal muscle fibres. J Cell Biol. 1983;97:756-71.

25. Gutman E, Carlson BM. Contractile and histochemical properties of regenerating crosstransplanted fast and slow muscles in the rat. Plugers Arch 1975;353:227-39. 